American Journal of Applied Sciences 7 (11): 1521-1527, 2010

ISSN 1546-9239

(C) 2010 Science Publications

\title{
Improving an Electrostatic Powder Coating Process via Signal to Noise Response Surface
}

\author{
Pongchanun Luangpaiboon \\ Department of Industrial Engineering, Faculty of Engineering, \\ Industrial Statistics and Operational Research Unit, \\ Thammasat University, Pathumthani, 12120 Thailand
}

\begin{abstract}
Problem statement: In a coating process, the master plate referenced brightness or color shade was critical to the exterior appearance on aluminum alloy wheels. The quality measures of interest are the metallic paint thickness values on four areas. Because of these lower quality levels, the manufacturer has spent more on the cost of restudy or scrap as well as the longer production time. Approach: An expert system revealed seven process variables affecting those quality characteristics. Taguchi signal to noise ratio or $\mathrm{SN}$ of paint thickness values on the outboard spoke area was determined as a process response whereas SN on the remaining areas of window outboard, window between spoke and inboard spoke became merely process constraints. A constrained response surface optimization method and a modified complex method were performed to move the current operating condition towards the optimum. Results: The new settings improved the paint thickness values in terms of both the average and the standard deviation on all critical areas except the window between spoke. Conclusion: As expected, the implementation brings the reduction of the metallic paint consumption and the mismatch level of color shade.
\end{abstract}

Key words: Constrained optimization, Modified Complex Method (MCM), mismatch level, Electrostatic Powder Coating Process (EPCP), Response Surface Methodology (RSM), Evolutionary Operation (EVOP), chemometrics, Modified Simplex Method (MSM), metallic paint thickness

\section{INTRODUCTION}

The selections of aluminum alloy wheels are based on the performance, the feature and the appearance. The first two are the perceived quality for each company. For the appearance, the color shade and paint durability are important since they are the first noticeable quality for the user. The case of interest has faced with the lower quality problem being complained by customers in both the metallic paint thickness and the master plate referenced brightness at the final quality gate of production. The first time quality level is low at $75 \%$ and this leads to the high production cost of restudy or scrap.

To decrease such problem, a system with electrostatic spraying guns is invested for a painting process and it is referred to as an Electrostatic Powder Coating Process (EPCP) (Fig.1). Powder coating is mainly applied for coating of metals, such as aluminum extrusions, medium-density fiberboard and automobile or bicycle parts. It is used to create a hard finish that is tougher than the conventional painting process. The selected machines or devices would provide a good appearance, good leveling rate of spraying, good metallic paint effect. With the high technology, the problem has still existed. In this case, the deep details of the EPCP should be investigated to determine the optimal operating condition.

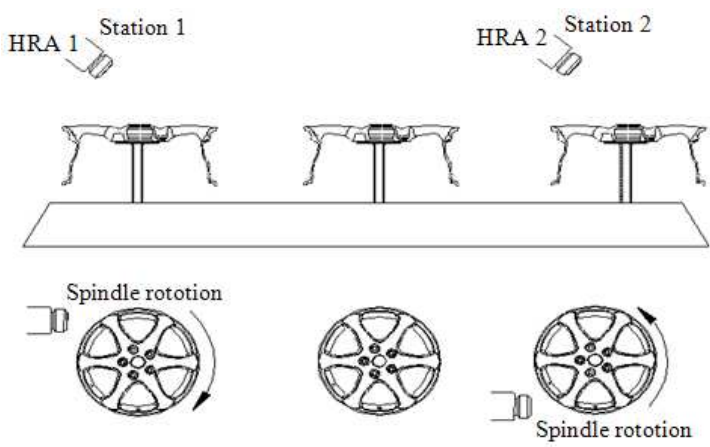

Fig. 1: Powder application process of the EPCP

Corresponding Author: Pongchanun Luangpaiboon, Department of Industrial Engineering, Faculty of Engineering, Thammasat University, Pathumthani, 12120, Thailand 
Am. J. Applied Sci., 7 (11): 1521-1527, 2010

Most manufacturing processes including the EPCP have some variables. These process variables can be adjusted by plant operators or by automatic control mechanisms to enhance the efficiency of the machine. Care must be taken to operate industrial processes within safe limits, but optimal conditions are rarely attained and increased international competition means that deviations from the optimum can have serious financial consequences. Usually the functional relationship between process variables and the yield is unknown. Response Surface Methodology (RSM) or Evolutionary Operation (EVOP) is then used to improve the current operating conditions of industrial processes.

Factorial experimental designs have been applied to determine the path of steepest ascent. The objective of evolutionary operation is to move a process towards optimum as quickly as possible. Many textbooks offer advices on this strategy. There is a theoretical argument that the minimal number of design points to estimate the direction of steepest ascent, from noisy observations, is just one more than the number of process variables for the optimization or the dimensions of the search space $(\mathrm{k})$ that is a simplex design (Farshi et al., 2011). For $\mathrm{k}$ equal to two, this simplex is an equilateral triangle composed of three vertices in the 2dimension space, for $\mathrm{k}$ equal to three it is a tetrahedron (Kamoun et al., 2009).

The rigid simplex algorithm was proposed for finding the process optimum (Luangpaiboon, 2009). The first cycle requires the response evaluation in each design point of the initial simplex. The design point with the lowest yield is reflected in the opposite face and the next run is carried out at this point. This idea is not commonly referred to in statistical texts but has received more attention in the chemometrics literature (Dong et al., 2009). They tried various rules for making the algorithm proceed more efficiently without an oscillation or rotating about a design point. These rules improved the algorithm to some extent, but a more significant variation was the Modified Simplex Method (Zhao et al., 2009) Instead of limiting the new vertex to the mirror image of the least vertex, an expansion or contraction of the reflection is allowed at each step. The complex method is an alternative among those to determine the optimal setting of process variables under a consideration of process constraints (Chen et al., 2010).

Previous studies applied not only two main classes of factorial and simplex designs, but they introduced Taguchi parameter designs to industries as well (Ismail et al., 2009). Choi et al. (2010) expressed wire-woven bulk Kagome (WBK) or a new truss-type cellular metal fabricated by assembling helically-formed wires. The
Taguchi method was used to minimize the number of experiments required to evaluate the sensitivity of each design parameter with the specific compressive strength of WBK. The height of the braze normalized by the wire diameter is the least influential design parameter. Various Taguchi parameter designs and performance measures were applied on many engineering problems and brought proper levels of operating conditions and expenses (Kumar and Khamba, 2008; Jailani et al., 2009; Huang and Shiau, 2009).

Nowadays there are some difficulties for using response surface optimizations on noisy, complex and large-scale problems (Srivastava and Srivastava, 2008). Various algorithms for the exploration of a dual response surface have been proposed. The aim is to search for variable settings which optimize a primary response (mean) function while simultaneously satisfying constraints of secondary response (variance) functions. Jeong et al. (2010) expressed how the goals of a dual response optimization approach can be achieved using standard non-linear programming technique, the generalized reduced gradient algorithm. It has been claimed that the proposed method leads to be more flexible and easier to use than the original dual response approaches. Costa (2010) also proposed a more satisfying and substantially simpler optimization procedure on a dual response approach.

Currently, product and process improvements need the flexibility and low expenses. Alternatives of experimental designs and evolutionary operations could be efficiently selected to determine the proper level of operating conditions. In this study, not only the best choices of Taguchi parameter design and two sequential procedures are applied to the electrostatic powder coating process, but the performance measure on both the sample mean and standard deviation in terms of the signal to noise ratio is also used as a process response instead of the conventional yield. On the preliminary study phase, the constrained response surface optimization model is performed to recheck the most influential process variables from an initial determination by an expert system. This research then undertakes further investigations with a sequential movement of the modified complex method to determine the proper levels of influential process variables for the EPCP.

\section{MATERIALS AND METHODS}

Electrostatic Powder Coating Process (EPCP): In the EPCP, the precisely atomized paint particles are made to be electrically charged by repelling each other and spreading themselves while exiting the spray nozzle. 
Am. J. Applied Sci., 7 (11): 1521-1527, 2010

The wheel being painted is then charged oppositely or grounded. The paint is attracted to the wheel giving a more even coat than the wet spray painting and also increasing the percentage of the paint that actually sticks to the wheel. This process means that the paint covers even hard to reach areas and the painting device (sprayer) with the unique application process causes the paint to be directly applied without overspray. Because the paint is magnetically drawn to the surface, items can be painted from one side and the paint will wrap around to the other side. The whole wheel is then baked to properly attach the paint, when the powder turns into a type of plastic.

By brainstorming from the teams who study for a coating shop, e.g., product and process engineers, maintenance operators, quality engineers and suppliers it has been summarized that there are seven process variables affecting the quality measures on aluminum alloy wheels. The first three process variables are related to the painting materials whereas the remaining process variables are from two electrostatic spraying guns of HRA1 and HRA2 at stations 1 and 2, respectively (Table 1 ).

All quality measures of interest on aluminum alloy wheels consist of the metallic paint thickness on four areas or zones of Z1-Z4 and the mismatch of color shade determined by the master plate referenced brightness. From the customer's specifications there are Lower (LB) and Upper (UB) bounds of metallic paint thickness (micron) on each zone as shown in Table 2. These provide the EPCP with four relevant responses to be optimized.

However, this study assigns the metallic paint thickness on $\mathrm{Z} 1$ as the primary or the most important response and others return to be merely secondary responses or problem constraints. The precise measurement system of the metallic paint thickness via a film measuring device can be determined by the $10 \%$ standard Gage R\&R level. During all phases of this study, \% Gage $\mathrm{R}$ and $\mathrm{R}$ is at 7.99 on average. The film measuring device has to be accepted throughout.

The color shade or brightness is measured by CHROMA METER CR-400/410. This process needs the master plate to compare and the difference or mismatch level is shown as the $\Delta \mathrm{E}$ value. It is related to the following parameters: L (a contrast of lightness or + versus darkness or -), a (a contrast of red or + versus green or - ) and $b$ (a contrast of yellow or + versus blue or -). If the $\Delta \mathrm{E}$ value is less than 1.0 the mismatch level of the color shade is accepted.
Table 1: Process variables of the EPCP

\begin{tabular}{ll}
\hline Process variables & Notations \\
\hline$x_{1}$ & Paint Resistance \\
$x_{2}$ & Electrostatic Charging \\
$x_{3}$ & Paint Viscosity \\
$x_{4}$ & Paint Flow Rate for HRA1 \\
$x_{5}$ & Ring Air Pressure for HRA 1 \\
$x_{6}$ & Paint Flow Rate for HRA 2 \\
$x_{7}$ & Ring Air Pressure for HRA 2 \\
\hline
\end{tabular}

Table 2: The customer's specifications of metallic paint thickness on each zone and its referenced numbers

\begin{tabular}{llll}
\hline \multicolumn{3}{c}{ each zone and its referenced numbers } \\
Zone & $\begin{array}{l}\text { Referenced } \\
\text { Zone }\end{array}$ & $\begin{array}{l}\text { Referenced } \\
\text { Number }\end{array}$ & $\begin{array}{l}\text { LB and UB } \\
\text { of thickness }\end{array}$ \\
\hline Z1 & Outboard & $19,21,23$, & $15-30$ \\
& Spoke & $25,27,29$ & \\
Z2 & Inboard & $20,22,24$, & $15-30$ \\
& Spoke & $26,28,30$ & \\
Z3 & Window & $1,3,4,6,7$, & $10-25$ \\
& Between & $9,10,12,13$, & \\
& Spoke & $15,16,18$ & \multirow{2}{*}{$10-25$} \\
Z4 & Window & $2,5,8,11$, & \\
& Outboard & 14,17 & \\
\hline
\end{tabular}

Taguchi design and analysis: Taguchi parameter design is an engineering strategy for the robust process or product (Zandieh et al., 2009). An aim is to search for variable settings that minimize the response variation while attaining the process or product on its target over various conditions. This also makes the product or process insensitive to the component deterioration or changes in the environmental variation during its usage. Taguchi parameter designs are set to measure process variables associated with the interactions with the greatest importance from a standard linear graph.

Consequence of qualities is then marked by a signal to Noise Ratio (SN), which will be used to point out the stability of the design system and quality of chosen design parameters (Pal and Gauri, 2010). The SN provides a standard index for the data comparison, while having a set of $n$ replicates. Based on the optimization nature, $\mathrm{SN}$ can be categorized into three classes of the smaller the better, the larger the better and the target the better. The designer then adjusts the levels of design parameters to achieve the goal of the experimentation via main effect and interaction graphs.

Methods: This study proposes the Modified Complex Method (MCM) to optimize the constrained signal to noise response surface for the EPCP. This variant is a hybridization of the Box complex and the Nelder and Mead modified simplex methods. The objective is to drive the process operating condition towards the region of the variable space which is of the optimal response with additional conditions of all satisfied constraints. 


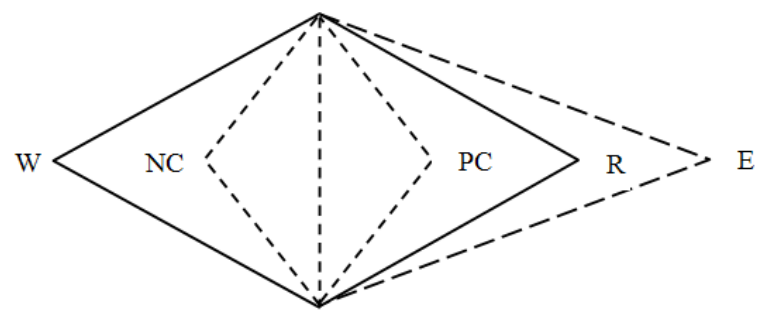

Fig. 2: Different Simplex Moves from the Rejected Trial Condition (W). $\mathrm{R}=$ Reflection, $\mathrm{E}=$ Expansion, $\mathrm{PC}=$ Positive Contraction and $\mathrm{NC}=$ Negative Contraction

In the complex method, the initial design points are randomly taken around a current operating condition. It is assumed that an initial design point of process variables which satisfies all the constraints is available. The initial form of these design points, which is called as a complex, is not necessary to be a precise geometric form. The complex is just a flexible mathematical form made up of at least $\mathrm{k}+1$ design points. The convex hull of the $\mathrm{k}+1$ design points that define a $\mathrm{k}$-simplex is called a face. During its sequential procedures the worst vertex is replaced by its symmetrical design point affected by some coefficients. After each step, a decision is made to determine in which direction the complex must move.

For the Modified Simplex Method (MSM), NelderMead modified the rigid simplex algorithm to allow various procedures to adapt to the response surface optimization much more readily. The MSM allows the simplex to converge more rapidly towards an optimum by expansion and multiple ways of simplex contraction along the line of conventional reflection in order to speed up the convergence (Fig. 2).

When the response at the reflected vertex is more preferable than the responses from all previous vertices, the expansion with a preset expansion coefficient is applied, to stretch the move. In some cases when the response at the reflected vertex is more desirable than the worst one, but still worse than all the remaining responses, the contraction with a preset contraction coefficient is applied to make the move shorten in comparison to the reflection. Moreover, massive contractions are applied when the new response gets worse than any of the previous ones. In this case the size of the simplex is reduced by contracting each of its edges to one half of its previous length toward the vertex producing the best response. These rules are repeated until the convergence criteria are met. The simplex moves over the response surface and should contract around the optimum.
The Modified Complex Method (MCM) begins with a preset number of design points (larger than $\mathrm{k}+1$ ) within feasible ranges of the lower and upper bounds of process variables. A design point selected must satisfy the explicit constraints of process variable levels, but need not satisfy all the implicit constraints of secondary responses. If an implicit constraint is violated or a design point is found to be infeasible, a new design point is modified by previously generated design points. To successfully improve the process, the MCM parameters need to be determined before applying its sequential procedures. They include the number of replicates in each design points and termination criteria of $\varepsilon$ and $\delta$ for the response difference and the size of the complex, respectively.

These stopping criteria built into the method are conservative that is the MCM shall stop itself when there is no improvement of consecutive evaluations. This implies the MCM will not terminate when there is any chance of further improvement in responses, but avoids the complex to be shrunk to such a size that changes in the response are smaller than a preset value. It is necessary that the feasible region should be convex or the optimum does not lie at a constraint boundary. Otherwise a retraction to the centroid may result in infeasible design points and the speed of the method tends to become somewhat slow due to a zigzag effect.

In this study iterative strategies of the MCM have $\mathrm{SN}$ of paint thickness values on the outboard spoke area $\left(\mathrm{Y}_{\mathrm{p}}\right)$ as a moving trigger whereas $\mathrm{SN}$ of paint thickness values on the remaining areas of window outboard, window between spoke and inboard spoke return to be merely process constraints or secondary responses $\left(\mathrm{y}_{\mathrm{s}}\right)$. The generation of the initial MCM begins with determining feasible initial design points that satisfy both explicit and implicit constraints. Implicit constraints limit the values of secondary responses and explicit constraints limit the values of process variables. The MCM parameters: the number of replicates of $n, a$ reflection coefficient of $\alpha_{R}$, an expansion coefficient of $\alpha_{\mathrm{E}}$, a positive contraction coefficient of $\alpha_{\mathrm{PC}}$, a negative contraction coefficient of $\alpha_{\mathrm{NC}}$ and termination criteria of $\varepsilon$ and $\delta$ for the response difference and the coded size of the complex are set at $5,1,2,0.5,-0.5,0.001$ and 0.05 , respectively. The iterations replicate until the termination criteria is at the satisfaction state. Whilst continually checking the termination criteria, following steps below would be carried out:

Step 1: Generate an initial set of $\mathrm{N}$ ample design points $\left(\mathrm{x}^{\mathrm{i}}\right)$ located possibly near the current operating condition within explicit and implicit constraints.

- If $\mathrm{x}^{\mathrm{i}}$ is infeasible, determine a centroid or $\mathrm{x}^{\mathrm{C}}$ of a current set of feasible design points and reset: $x^{i}=x^{i}+1 / 2\left(x^{C}-x^{i}\right)$ 
- Evaluate the response of $y_{P}$ from $n$ replicates in each feasible design point $\left(\mathrm{x}^{\mathrm{i}}\right)$

Step 2: Calculate the new design point $\left(\mathrm{x}^{\mathrm{MCM}}\right)$ from the ordinary MSM motions.

- If $\mathrm{x}^{\mathrm{MCM}}$ is feasible, go to Step 3

- If $\mathrm{x}^{\mathrm{MCM}}$ is infeasible, go to check for the solution feasibility of the MCM. For each i, reset violated variable bounds: If $\mathrm{x}_{\mathrm{i}}^{\mathrm{MCM}}<\mathrm{x}_{\mathrm{i}}^{\mathrm{L}}$ set $\mathrm{x}_{\mathrm{i}}^{\mathrm{MCM}}=\mathrm{x}_{\mathrm{i}}^{\mathrm{L}}$ If $x_{i}^{\mathrm{MCM}}>\mathrm{x}_{\mathrm{i}}^{\mathrm{U}}$ set $\mathrm{x}_{\mathrm{i}}^{\mathrm{MCM}}=\mathrm{x}_{\mathrm{i}}^{\mathrm{U}}$

- If the resulting $\mathrm{x}^{\mathrm{MCM}}$ is still infeasible, retract half the distance to the centroid, $\mathrm{x}^{\mathrm{MCM}}=\left(\mathrm{x}^{\mathrm{C}}+\right.$ $\left.\mathrm{x}^{\mathrm{MCM}}\right) / 2$. Continue until $\mathrm{x}^{\mathrm{MCM}}$ is feasible and go to Step 3

Step 3: An implementation will be carrying out to measure the primary response of $y_{P}$. Check for the MCM termination rules below. If both rules do not meet, continue and go to Step 2:

$$
\begin{aligned}
& \text { - Calculate } \bar{y}_{\mathrm{p}}=\frac{1}{\mathrm{~N}} \sum_{\mathrm{i}} \mathrm{y}_{\mathrm{p}}\left(\mathrm{x}^{\mathrm{i}}\right) \text { and } \mathrm{x}^{\mathrm{c}}=\frac{1}{\mathrm{~N}} \sum_{\mathrm{i}} \mathrm{x}^{\mathrm{i}} \\
& \text { - If } \sqrt{\sum_{\mathrm{i}}\left(\mathrm{y}_{\mathrm{p}}\left(\mathrm{x}^{\mathrm{i}}\right)-\overline{\mathrm{y}}_{\mathrm{p}}\right)^{2}} \leq \varepsilon \text { and } \sqrt{\sum_{\mathrm{i}}\left(\mathrm{x}^{\mathrm{i}}-\mathrm{x}^{\mathrm{c}}\right)^{2}} \leq \delta
\end{aligned}
$$

\section{RESULTS}

As stated before, there are seven process variables affecting the quality measures in the EPCP. Moreover, the relationships on those process variables in terms of two variable interactions provide eight selected interactions with the greatest importance. If there are two levels in each process variable the most suitable orthogonal array of all six types of Taguchi parameter designs matches the standard Taguchi linear graph for L16 $\left(2^{15}\right)$ of type II. During all phases of experiments, the specific standard performance levels of the machines and devices for all remaining trivial process variables; conveyor speed, rotation speed, directions of the wheel at stations 1 and 2, rotor speed, formed air pressure, temperature, relative humidity and circulated air flow in spray booth were set at $4.1 \mathrm{~m} \mathrm{~min}^{-1}, 40 \mathrm{rpm}$, clockwise, counter-clockwise, $28000 \mathrm{rpm}, 1.3$ bars, 2530 Celsius, $65-85 \%, 26550-29500 \mathrm{~m}^{3} \mathrm{~h}^{-1}$, respectively.

On a preliminary study, all the experimental runs from the orthogonal array of L16 were used to analyze the regression coefficients of $y_{p}$ and $\hat{y}_{s}$. These estimates were measured to compare with the approximate $95 \%$ confidence interval. A Linear Constrained Response Surface Optimization Model (LCRSOM) was then formulated to find the process variable setting with the highest level of the estimated primary response of $\dot{y}_{p}$ and satisfying all other constraints of Estimated
Secondary Responses $\left(\dot{y}_{\mathrm{s}}\right)$. Moreover, Lower (LB) and Upper (UB) bounds of process variables (x) were included in order to avoid solutions that extrapolate too far outside the feasible region of the experimental design points, namely:

$$
\begin{aligned}
& \text { Optimize } \grave{y}_{\mathrm{p}} \\
& \text { Subject to } \\
& \qquad \mathrm{LB} \leq \mathrm{y}_{\mathrm{s}} \leq \mathrm{UB} \\
& \mathrm{LB} \leq \mathrm{x} \leq \mathrm{UB}
\end{aligned}
$$

Consider the model fit above, the new estimated design points, achieved by the generalized reduced gradient algorithm, was $\mathrm{x}_{1}=100, \mathrm{x}_{4}=70, \mathrm{x}_{5}=2.0$ and $\mathrm{x}_{6}=70$ whereas remaining process variables were similar to the current operating condition. This new operating condition brought the reduction of the mismatch level of color shade from 2.91-0.92 and the metallic paint consumption at the acceptable level of 56 grams or a decrease for $4 \mathrm{~g}$ in each alloy wheel. The metallic paint thickness on each zone was within the customer's specification except Z3 or the area of window between spoke which is the most difficult area to spray all over that region. It could be enhanced by increasing the combination of pigments and resins, though this brings higher level of expense, or applying other sequential response surface algorithms.

Based on at least two significant effects on primary or secondary responses, the MCM with five process variables was considered in order to decrease the number of design points. There were ten initial design points needed for the process improvement on the next phase. The feasible design points were then measured the primary responses. The worst design point was then found and reflected over the centroid of the remaining design points. In the first iteration the MCM with the expansion provided the new design point of $x_{1}=92, x_{2}$ $=55, x_{4}=72, x_{6}=74$ and $x_{7}=2.6$ whereas other process variables were fixed at the same levels from the CRSOM operating condition. The experimental results showed that the metallic paint thickness on each zone was still within the customer's specification, except Z3.

In the second iteration, the new design point from a positive contraction was infeasible, before we did any modification, a check of whether the obtained design point was close to a variable boundary or not was performed. Since $\mathrm{x}_{1}{ }^{\mathrm{MCM}}>\mathrm{x}_{1}{ }^{\mathrm{U}}$ set $\mathrm{x}_{1}{ }^{\mathrm{MCM}}=\mathrm{x}_{1}^{\mathrm{U}}$, which did lie on the boundary. This resulting $\mathrm{x}^{\mathrm{MCM}}$ design point was still infeasible on all secondary responses, we moved to Step 2c). We retracted half the distance to the centroid $\left(\mathrm{x}^{\mathrm{C}}\right)$ and the new design point of $\mathrm{x}^{\mathrm{MCM}}$ was $\mathrm{x}_{1}$ $=75, x_{2}=60, x_{4}=67, x_{6}=67$ and $x_{7}=2.8$. 
Am. J. Applied Sci., 7 (11): 1521-1527, 2010
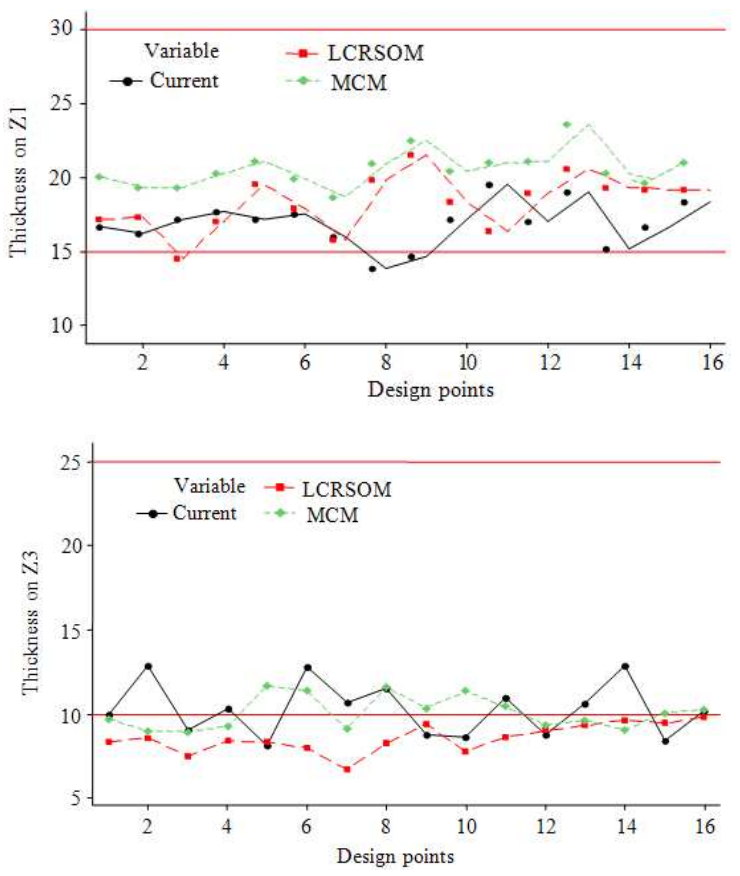

Fig. 3: Patterns of the metallic paint thickness including lower and upper bounds of the customer's specifications on $\mathrm{Z} 1$ and $\mathrm{Z} 3$

At this design point, the constraints were not violated, thus the design point was feasible. This MCM design point was set as a new operating condition and moved to Step 3).

The experimental results on paint thickness values repeated the same quality performances on all zones. However, the levels of both mean and standard deviation of paint thickness values on the outboard spoke area (Z1) seemed superior to the previous levels. Though there was no quality improvement on the average metallic paint thickness on the window between spoke area (Z3), the variation decreased as expected via the concept of Taguchi signal to noise ratio (Fig. 3).

\section{DISCUSSION}

The EPCP: The response surface methods will generally search for the proper operating conditions under a consideration of a uni-response system. In this study, the quality measures of interest are the metallic paint thickness values on four zones including the master plate referenced brightness or color shade. This study then deals with multiple responses. Taguchi signal to noise ratio is applied as a process response instead of the actual process yield. The EPCP uses automatic spraying guns and there are seven process variables that affect the quality measures as mentioned above.
Table 3: A Comparison of process variable levels and their quality measures from the Linear Constrained Response Surface Optimization Method (LCRSOM) and Modified Complex Method (MCM)

\begin{tabular}{llll}
\hline & & & Operating conditions \\
Process & Current & LCRSOM & MCM \\
Variables & 90 & 100 & 75 \\
\hline $\mathrm{X}_{1}$ & 50 & 50 & 60 \\
$\mathrm{X}_{2}$ & $9.5-10.0$ & $9.5-10.0$ & $9.5-10.0$ \\
$\mathrm{X}_{3}$ & 75 & 70 & 67 \\
$\mathrm{X}_{4}$ & 2.3 & 2.0 & 2.0 \\
$\mathrm{X}_{5}$ & 75 & 70 & 67 \\
$\mathrm{X}_{6}$ & 2.5 & 2.5 & 2.8 \\
$\mathrm{X}_{7}$ & 60 & 56 & 52 \\
Paint & & & \\
Consumption & 32 & 30 & 26 \\
via HRA 1 & 28 & 26 & 26 \\
via HRA 2 & & & \\
Brightness & 79.96 & 81.96 & -0.59 \\
$\mathrm{~L}$ & -0.61 & -0.62 & -0.45 \\
$\mathrm{a}$ & -0.32 & -0.32 & 0.82 \\
$\mathrm{~b}$ & 2.91 & 0.92 & \\
$\Delta \mathrm{E}$ & & & \\
\hline
\end{tabular}

The LCRSOM and MCM: The new levels of process variables bring the $13 \%$ reduction on the metallic paint usage in each wheel during the manufacturing process and improve the first time quality from $75 \%$ to $88 \%$. The mismatch level of color shade $(\Delta \mathrm{E})$ determined by the master plate referenced brightness $(\mathrm{L}=82.86, \mathrm{a}=$ 0.67 and $b=-0.54$ ) is less than 1.0 which is better than the previous operating condition (Table 3). As stated earlier, the experiments in this research were restricted to two iterations. Consequently the new operating condition might not be the optimum and further investigations are needed to improve the process in a context of the response surface methodology. Other stochastic approaches could be extended to the method based on conventional factorial designs to compare its performance, especially in terms of speed of convergence and when the error standard deviation of the process is at higher levels.

\section{CONCLUSION}

The electrostatic powder coating process creates a durable finish on the metal. Like traditional liquid paints some plastics without the runs, drips and bubbles can occur via the principle of the opposite attract. Concepts and operations of the EPCP are simple. Dry powder with the combination of pigments and resins is pneumatically fed from a supply reservoir to an electrostatic spraying gun where the high voltage charge is imparted to the powder. To be finished the part is electrically grounded. When the charged powder particles are sprayed, they are firmly attracted to the grounded part surface. They are held there until melted and fused into a smooth coating in the curing ovens. 
Though there are some successful levels of process variables from different circumstances of the EPCP, they seem impractical for painting aluminum alloy wheels in an automotive industry. Optimized parameter settings via signal to noise response surface methods improved the paint thickness at the critical position and satisfied the remaining specifications.

\section{ACKNOWLEDGMENT}

This study was supported by the Thailand Research Fund (TRF), the National Research Council of Thailand (NRCT) and the Commission on Higher Education of Thailand.

\section{REFERENCES}

Chen, G., L. Tang and Z. Li, 2010. Parameter optimisation of human-simulated intelligent controller for a cart-double pendulum system. Int. J. Modell. Identific. Control, 10: 194-201. DOI: 10.1504/IJMIC.2010.034571

Choi, J.E, G.D. Ko and K.J. Kang, 2010. Taguchi method-based sensitivity study of design parameters representing specific strength of wirewoven bulk Kagome under compression. Composite

92: Struct., 10.1016/j.compstruct.2010.01.024

Costa, N.R.P., 2010. Simultaneous optimization of mean and standard deviation. Quality Engineer., 22: 140-149. DOI: 10.1080/08982110903394205

Dong, X., C.A. Mattingly, M. Tseng, M. Cho and V.R. Adams, 2009. Development of new lipidbased paclitaxel nanoparticles using sequential simplex optimization. Eur. J. Pharm. Biopharm., 72: 9-17. DOI: 10.1016/j.ejpb.2008.11.012

Farshi, B., S. Gheshmi and E. Miandoabchi, 2011. Optimization of injection molding process parameters using sequential simplex algorithm. Mat. Design, 32: 414-423. DOI: 10.1016/j.matdes.2010.06.043

Huang, Y.M. and C.S. Shiau, 2009. An optimal tolerance allocation model for assemblies with consideration of manufacturing cost, quality loss and reliability index. Assembly Automat., 29: 220-229. DOI: 10.1108/01445150910972903

Ismail, A.R., M.R.A. Rani, Z.K.M. Makhbul, K. Sopian and B.M. Deros, 2009. Thermal comfort assessment and optimization of environmental factors by using Taguchi method. Am. J. Applied Sci., $\quad 6: \quad 1731-1741 . \quad$ DOI: 10.3844/ajassp.2009.1731.1741
Jailani, H.S., A. Rajadurai, B. Mohan, A.S. Kumar and T. Sornakumar, 2009. Multi-response optimization of sintering parameters of Al-Si alloy/fly ash composite using Taguchi method and grey relational analysis. Int. J. Adv. Manufact. Technol., 45: 362-369. DOI: 10.1007/s00170-009-1973-3

Jeong, I.J., K.J. Kim and D.K.J. Lin, 2010. Bayesian analysis for weighted mean-squared error in dual response surface optimization. Quality Reliabil. Engineer. Int., 26: 417-430. DOI: $10.1002 / q r e .1058$

Kamoun, A., M. Jaziri and M. Chaabouni, 2009. The use of the simplex method and its derivatives to the on-line optimization of the parameters of an injection molding process. Chemom. Intel. Laboratory Syst., 96: 117-122. DOI: 10.1016/j.chemolab.2008.04.010

Kumar, V. and J.S. Khamba, 2008. Statistical analysis of experimental parameters in ultrasonic machining of tungsten carbide using the taguchi approach. J. Am. Ceramic Soc., 91: 92-96. DOI: 10.1111/j.1551-2916.2007.02107.x

Luangpaiboon, P., 2009. Hybridizations of simulated annealing and modified simplex algorithms on a path of steepest ascent with multi-response for optimal parameter settings of ACO. IAENG Trans. Engineer. Technolog., 3: 94-108. DOI: 10.1063/1.3256264

Pal, S. and S.K. Gauri, 2010. Multi-response optimization using multiple regression-based weighted signal-to-noise ratio (MRWSN). Qual. Engineer., $\quad 22: \quad 336-350 . \quad$ DOI: $10.1080 / 08982112.2010 .495368$

Srivastava, R. and R.K. Srivastava, 2008. Optimization of process parameters in solid lubricant (MoS2) assisted machining of AISI 1040 steel by response surface methodology. Int. J. Machin. Machinabil. Mater., $\quad 4: \quad 111-128 . \quad$ DOI: 10.1504/IJMMM.2008.020914

Zandieh, M., M. Amiri, B. Vahdani and R. Soltani, 2009. A robust parameter design for multi-response problems. J. Comput. Applied Mathemat., 230: 463-476. DOI: 10.1016/j.cam.2008.12.019

Zhao, Q.H., D. Urosević, N. Mladenović and P. Hansen, 2009. A restarted and modified simplex search for unconstrained optimization. Comput. Operations Res., 36: 3263-3271. DOI: 10.1016/j.cor.2009.03.005 